\title{
Ion Beam Probe for Measurement of Wiggler Errors
}

\author{
John Vetrovec, Bradley Bobbs, and Michael Lampel \\ Rockwell International, Rocketdyne Division \\ 6633 Canoga Ave. \\ Canoga Park, Calif. 91304, (818) 700-4875
}

Abstract

Precise characterization of wiggler field errors is needed in order to perform compensations. Traditional method, which relies solely on Hall probe data acquired from a field map, suffers due to cumulative noise effects and is inadequate for long wigglers. The proposed instrument avoids this problem by directly measuring an equivalent trajectory of a low energy ion beam which is momentum and emittance matched to the electron beam. The ion beam is injected into the wiggler where it is imaged onto the screen of a miniature CCD camera which travels through the wiggler bore. By combining data from the camera and the Hall probe, the instrument achicves a resolution superior to a Hall probe alone.

\section{INTRODUCTION}

The Ground Based Free Elcctron Laser Technology Integration Experiment (GBFEL-TIE) in White Sands, NM, calls for a wiggler 400 periods long. Strong concerns exist over the cumulative effects of wiggler field errors on the trajectory and the phase of the electron beam. Several correction schemes using 20 pairs of beam position monitors (BPMs) and steering correctors are being considered [1]. However, BPMs provide only a discrete sampling (every 20 periods) of the trajectory at a rather low resolution (100 $\mu \mathrm{m}$ ). Since this data appears insufficient for wiggler tuning, other, more reliable means for trajectory analysis are sought. This article describes the Ion Beam Probe System (IBPS) which promises to provide reliable trajectory measurements.

\section{INSUFFICIENCY OF HALL PROBE DATA}

Traditionally, the magnetic field of the wiggler is mapped by a Hall effect probe and this data is used to reconstruct the electron beam trajectory, which, in turn, is used to tune wiggler taper and steering correctors. While this technique is entirely sufficient for short wigglers, accumulation of the residual Hall probe sampling errors (largely due to mechanical alignment of the probe) along the 400 -period wiggler becomes intolerable. The most severely affected is the estimate of the phase of the electron motion with respect to the optical wavefronts. As the FEL performance shows a strong correlation to the phase [2], this problem is of major importance.

\section{ION BEAM PROBE SYSTEM (IBPS)}

Accuracy of phase error measurements can be greatly improved with the proposed IBPS. Instead of reconstructing electron beam trajectory solely from Hall probe data, this instrument directly measures the trajectory as simulated by a beam of low energy ions having the same momentum as the electrons. Ions are injected into the wiggler bore where they are intercepted by a fluorescent screen on a CCD array mounted in a movable carriage (Figure 1). At the same time a pilot laser beam from a stable source is injected coaxially in to the wiggler and used as an alignment reference. Transverse position of the ion beam is then determined as a separation of the two beam spots viewed by the CCD chip (Figure 2). As the carriage is moved through the wiggler bore, the entire ion beam trajectory is resolved.

While the spatial resolution of the CCD limited by the noise in the optical signals to about $10 \mu \mathrm{m}$, the error in measured transverse position is not cumulative. Still, the raw trajectory data is too noisy to be useful for phase calculations, and must be locally smoothed. This process makes use of complementary Hall probe data also obtained by the instrument [3], thus combining the best qualities of the two data sets: global accuracy of the ion trajectory detector and local accuracy of the Hall probe. This reduces error accumulation and produces more reliable data which can be used for

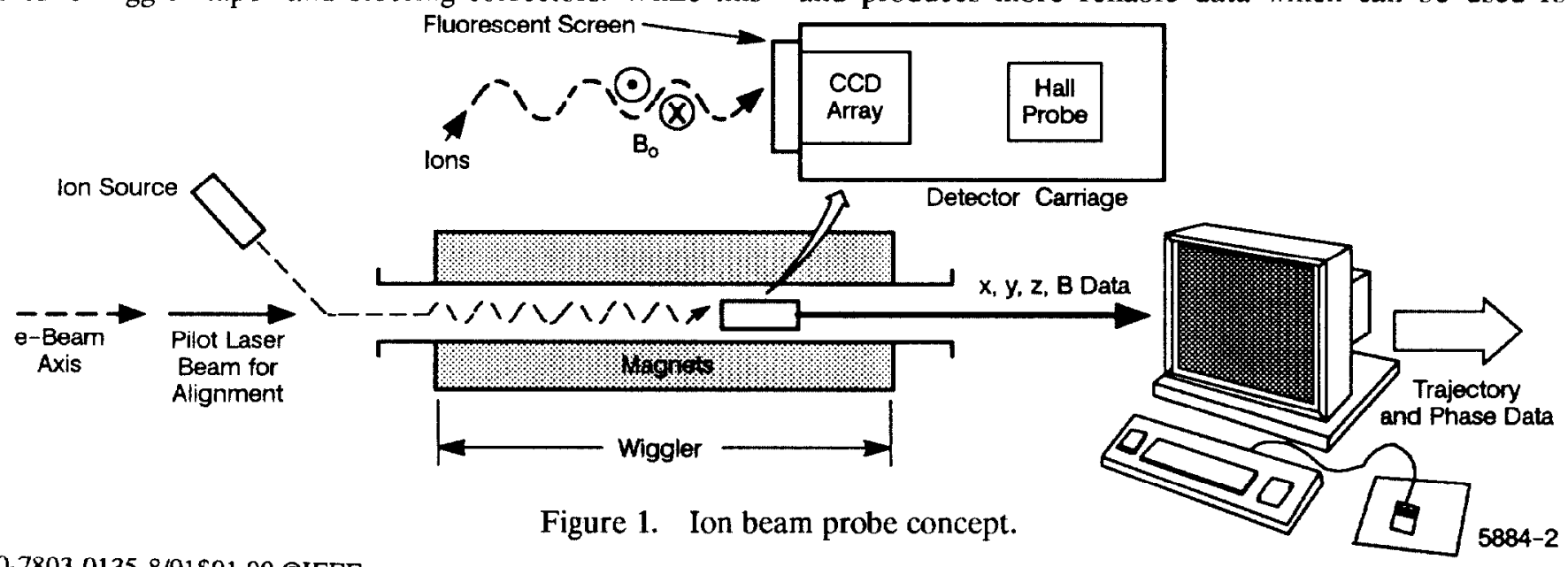


wiggler tuning, thereby improving the performance of the FEL.

The electron-ion momentum matching condition leads to a trade between ion mass and acceleration voltage. Required ion energy $T_{i}$ can calculated as $T_{i}=T_{e} m_{e} / M_{i}(\gamma / 2+1)$ where $T_{e}$ is the electron energy, $m_{e} / M_{i}$ is the ratio of electron and ion (rest) masses, and $\gamma$ is the relativistic factor. Since heavy ions have the least acceleration voltage requirements, the most abundant $\mathrm{Xe}(132)$ isotope was selected as a working specie. At the design electron beam energy of $100 \mathrm{MeV}$, momentum matched singly charged xenon ions are required to have an energy of only $41 \mathrm{keV}$.

In order to produce an ion beamlet of the same size as the electron beam, the ion beam emittance is matched by apodizing. Unfortunately, this also reduces the available beam current and the optical signal at the CCD. Although ncgative ions would follow exactly the same trajectory as clectrons, negative ion sources typically produce a substantially lower current than their positive counterparts. As a result, the DanFysik positive ion source model 920 was selected [4]. This device is capable of extracting a $10 \mathrm{~mA}$ xenon beam of $15 \pi \mathrm{mm}-\mathrm{mr}$ rms emittance through a $10 \mathrm{~mm}$ diameter aperture. While positive ions follow a trajectory which is a mirror image of the electron trajectory, this data contains all the necessary information to calculate the phase. A $45 \mathrm{deg}$ bending magnet working in conjunction with a small defining aperture is used to filter out unwanted xenon isotopes and

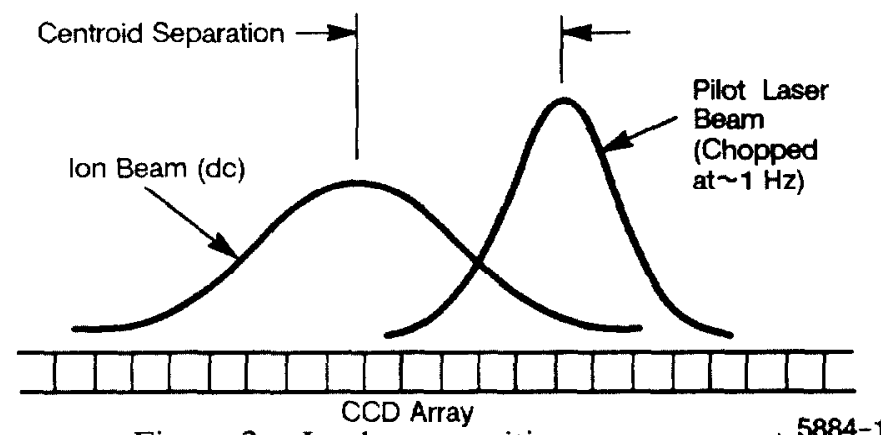

Figure 2. Ion beam position measurement. ${ }^{5884-1}$ other impurities. The aperture is sized to extract a beamlet with (unnormalized) emittance of $0.046 \pi \mathrm{mm}-\mathrm{mr}$ which, inside the wiggler, results in a beam spot diameter of about $1 \mathrm{~mm}$.

Inside the wiggler the ion beamlet is intercepted by a detector slowly moving through the bore. A possible detector is the Texas Instrument TC210 $196 \times 165$ planar CCD array with approximate pixel size of about $14 \mu \mathrm{m}$. This sensor is specifically packaged to allow mounting on a tip of small cross-section insertion probe. A layer of phosphorus coated over the protective glass window of the CCD sensor converts the kinetic energy of incident ions into an optical signal. Current density of the ion beam is about $65 \mathrm{nA} / \mathrm{mm}^{2}$, which is roughly the same as in a conventional cathode ray tube (CRT). It is somewhat conservatively assumed that using available phosphorus material the conversion efficiency of $41 \mathrm{keV}$ ions into visible spectrum light is about 100 times less effective then for 5-10 keV electrons (such as in a CRT). The intensity of light produced by the phosphorus screen is then about $0.032 \mu \mathrm{W} / \mathrm{mm}^{2}$. The CCD array has a typical 1000:1 dynamic range and, when scanned at $30 \mathrm{~Hz}$, it saturates at about $0.08 \mu \mathrm{W} / \mathrm{mm}^{2}$. This leads to about $400: 1$ signal to noise ratio, which appears quite satisfactory. In practice, the optimum signal-to-noise ratio in the CCD can be found by adjusting the scan rate.

\section{OPERATIONAL CONSIDERATIONS}

The IBPS is designed to operate in situ as a part of the electron beam line. It collects data needed to tune the wiggler prior to each FEL run. When inoperational, the detector carriage is hidden in an alcove at the downstream end of the wiggler (Figure 3 ). Radiation shield protects the CCD and Hall probe sensors from destruction when the electron beam is on. Since the alcove is evacuated, the carriage can be inserted into the wiggler without a vacuum break. The insertion process as well as the travel through the wiggler is under computer control. The carriage is mechanically propelled by a system of pulleys and wires hidden in longitudinal slots extruded into the vacuum tube bore. Longitudinal

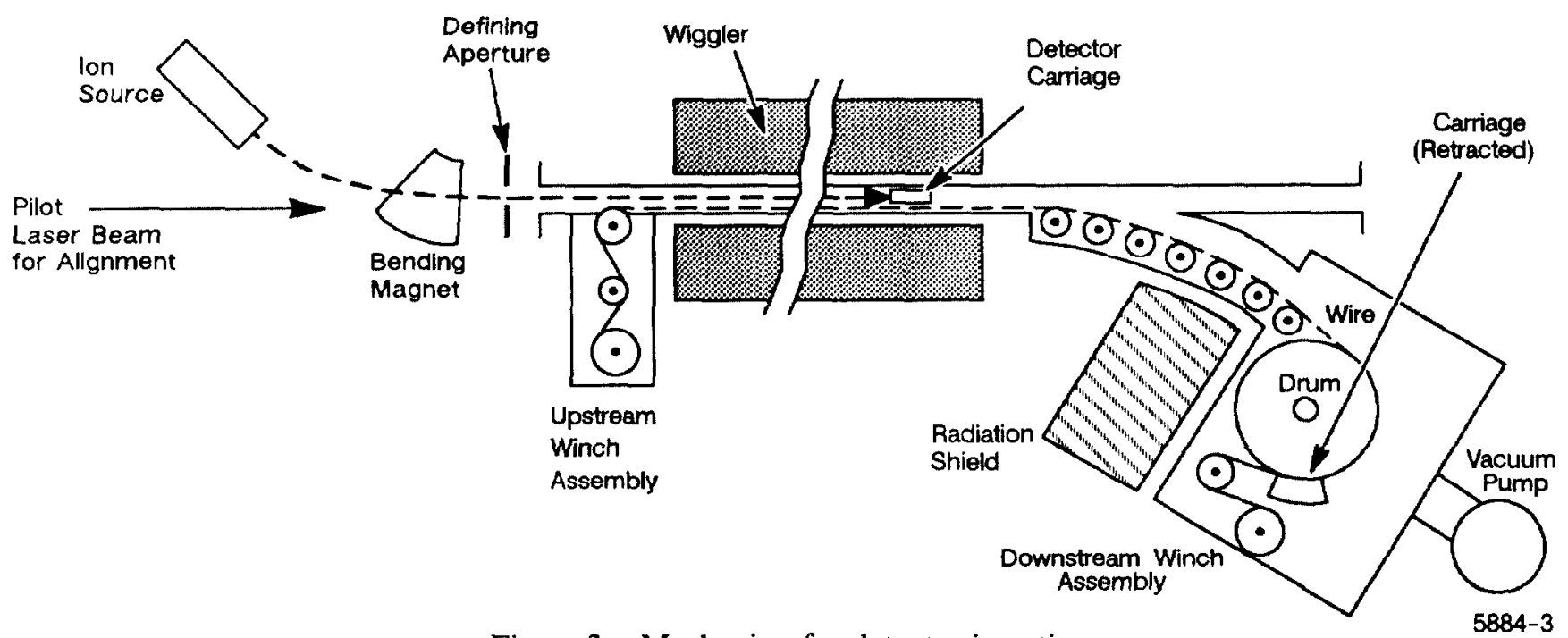

Figure 3. Mechanism for detector insertion. 
slots extruded into the vacuum tube bore. Longitudinal position of the carriage is inferred from a magnetic field profile monitored by a the Hall sensor contained within the carriage. This technique avoids the complexity of alternate mechanical or optical schemes while providing an accuracy of about 100 th of wiggler wavelength.

The detector carriage also contains the drive and the output signal electronics for both the CCD and the Hall sensor. The magnetic field of the wiggler is understood to have negligible impact on the function of the carriagemounted electronics. The CCD video signal with a bandwidth 1 to $10 \mathrm{MHz}$ (depending on the scan rate) is transmitted from the carriage to an external processing unit via $75 \mathrm{ohm}$ coaxial cable wound on the same drum as the motion wires. After digitizing, the CCD image is subjected to standard image processing techniques to determine the transverse position of the ion beam. Typically, 10-20 samples per wiggler period are taken. These data together with the I Iall probe signal are sufficient to reconstruct the local trajectory of the ion beam.

\section{SIMULATED PHASE MEASUREMENTS}

Performance of the IBPS has been evaluated using a one-dimensional model of the wiggler field and a particle transport simulator. General parameters are set to reflect the GBFEL-TIE wiggler design. Local magnetic field errors with the cosine half-wave spatial form suggested by Kincaid [5] are generated automatically by the computer to conform to a statistical model with Gaussian distribution. Field errors are unique to each run. Spatial resolution of the CCD system is primarily limited by the noise in the optical signals and it is conservatively assumed to be $10 \mu \mathrm{m}$. It is slightly less than 1 pixel size, and it represents about $20 \%$ of the wiggle amplitude $K /\left(k_{w} \gamma\right)$, where $K$ is the wiggler parameter and $k_{w}$ is the wiggler wave number.

An example computer run (Figure 4) shows the trajectories and phase retardations as calculated from the Hall probe data alone, from the IBPS data, and the actual (synthesized, error-free) conditions for the first 100 periods of the wiggler. Transverse displacement of the electron beam centroid measured by the ion beam sensor is shown before spatial smoothing. Clearly, despite a significant $(10 \mu \mathrm{m})$ local noise, the trajectory detected by the CCD array system faithfully follows the actual path. After smoothing, the two curves almost coincide. However, the trajectory calculated from Hall probe data alone drifts away after about 60 periods. Phase profile along the wiggler calculated from a smoothed trajectory also shows a good agreement with the actual data, while that based on Hall probe data exhibits large deviations.

\section{CONCLUSION}

The ion beam probe system promises to be a very accurate instrument which can be useful both during the initial commissioning as well as operational maintenance of the wiggler. Data obtained by the IPBS permits fine tuning of the wiggler and optimization of FEL performance. In addition, as the tuning is completed before the electron beam is injected, electron beam transport risk is reduced.

\section{ACKNOWLEDGMENTS}

A similar, but simpler, ion beam probe was used earlicr at LLNL to analyze field errors in the $5 \mathrm{~m}$ sections of the Paladin wiggler before installation into the ATA beam line. We wish to thank to H. Shay, B. Kulke, and G. Deis for providing us with information about the LLNL device.

\section{REFERENCES}

[1] C.J. Elliott, B.D. McVey, and D.C. Quimby, "Field Error Lottery," in proc. from the 12th Intl. FEL Conf., Paris, September 1990, North-Holland Elsevier, 1991, also to appear in Nucl. Instr. and Meth., 1991.

[2] J. Vetrovec, "Performance Model for FEL Wigglers," in proc. from the 12th Intl. FEL Conf., Paris, September 1990, North-Holland Elsevier 1991, also to appear in Nucl. Instr. and Meth., 1991.

[3] B. Bobbs and J. Vetrovec, "Characterizing Wiggler Phase Errors with an Ion Beam Probe," paper TC.7, in proc. from the Lasers ' 90 Conference, San Diego, CA, December 1990.

[4] High Current Ion Source Model 920 data sheets, DanFysik A/S, DK-4040 Jyllinge, Denmark, also available from GMW Associates, Redwood City, CA 94064.

[5] B. Kincaid, "Random Errors in Undulators and their Effect on the Radiation Spectrum," J. Opt. Soc. Am. B/Vol. 2, No. 8, pp. 1294-1306, August 1985.

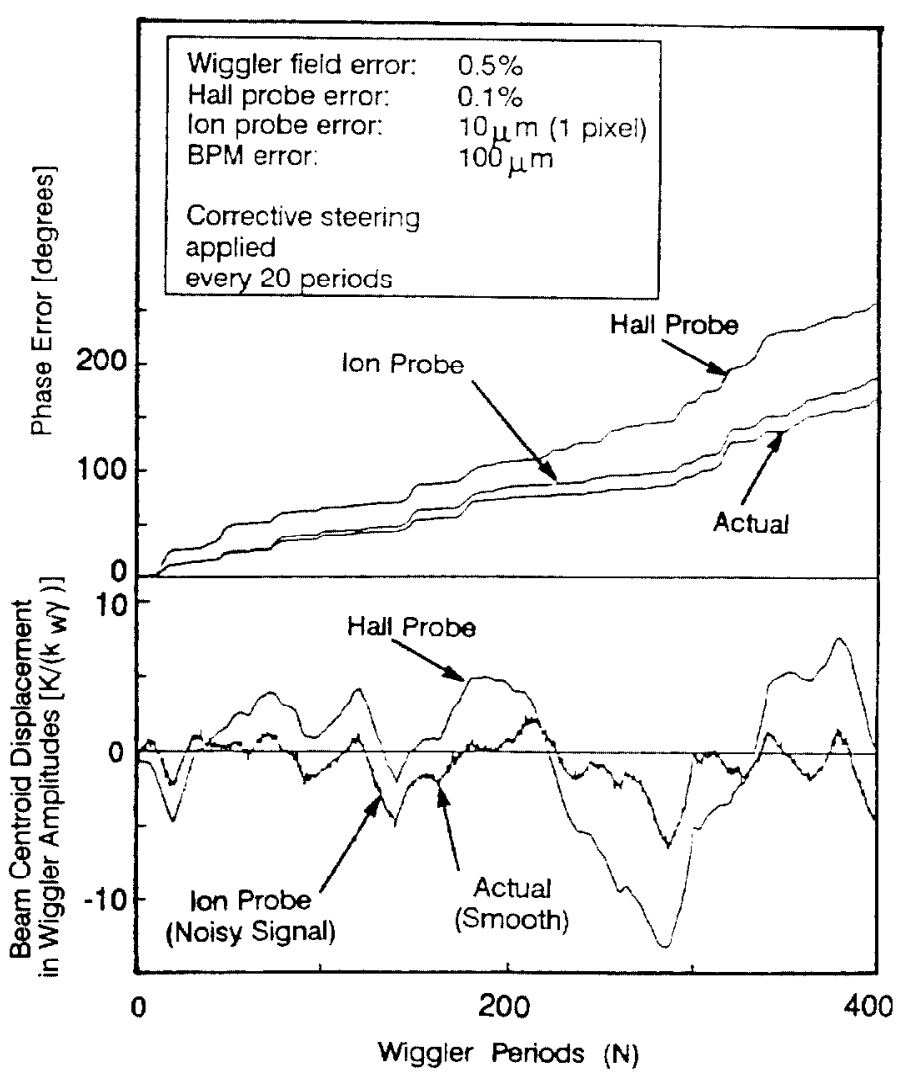

Figure 4. Comparison of measurements obtained by Ion and Hall probes (based on 1-D computer simulations). 